\title{
Exploración de variables predictoras de preñez en vaquillas de carne
}

\author{
Prieto, P.N.; Stahringer, C.R.; Vispo, P.E. \\ Instituto Nacional de Tecnología Agropecuaria (INTA), EEA Colonia Benítez \\ (Chaco, Argentina), Tel. 0369-4493054, E-mail: pprieto@correo.inta.gov.ar.
}

\begin{abstract}
Resumen
Prieto, P.N.; Stahringer, C.R.; Vispo, P.E.: Exploración de variables predictoras de preñez en vaquillas de carne. Rev. vet. 25: 1, 50-53, 2014. Con el objetivo de analizar variables pre-servicio que sean indicativas del desarrollo y la composición corporal, para encontrar aquéllas que sean predictivas de la tasa de preñez, se trabajó con un rodeo de 58 vaquillas en un establecimiento ganadero del nordeste argentino, donde se midieron diversas variables pre-servicio. A través de un modelo de regresión logística de todas las variables ensayadas como predictoras de preñez en dichas vaquillas, la altura a la grupa (ALT) y el score genital (SG) ajustaron al modelo de regresión. Si bien el peso vivo pre-servicio no logró un ajuste por sí mismo, se observó una fuerte asociación positiva entre éste y el SG. El modelo desarrollado permite predecir con una exactitud del $68 \%$ el resultado del servicio en las vaquillas (preñada o vacía). En las condiciones en que se desarrolló la experiencia, las vaquillas no deben superar los $122 \mathrm{~cm}$ de ALT y presentar un SG de 5 al inicio de la estación reproductiva para lograr la mayor probabilidad de preñez.
\end{abstract}

Palabras clave: vaquillas, predicción desempeño reproductivo.

\begin{abstract}
Prieto, P.N.; Stahringer, C.R.; Vispo, P.E.: Exploring variables associated with body reserves to predict pregnancy in beef heifers. Rev. vet. $25: 1,50-53$, 2014. In order to analyze variables that are indicative of body development and composition before breeding and to find out which of them could be predictive of pregnancy, this assay included 58 heifers from a cattle farm of North-eastern Argentina. From all the tested variables by means of a logistic regression model as predictors of pregnancy in heifers, the hip height (HH) and the reproductive tract score (RTS) fitted the regression model. Body weight (BW) before breeding did not adjust to the developed regression model. There was a strong positive association between BW and RTS. The model can predict with $68 \%$ of accuracy the result of service in heifers (pregnant or empty). Heifers must not exceed $122 \mathrm{~cm}$ in HH and present a RTS of 5 at the beginning of the breeding season to achieve the highest probability of pregnancy.
\end{abstract}

Key words: heifer, prediction of reproductive performance.

\section{INTRODUCCIÓN}

La tasa de crecimiento y la tasa reproductiva asumen gran importancia en producción animal debido a que el peso al destete y número de la progenie por vientre representan los mejores indicadores de eficiencia en un sistema ganadero. Tales sistemas están condicionados por la precocidad en el inicio de la actividad reproductiva de la vaquilla y por el retorno temprano al celo en la vaca postparto (PP) ${ }^{4}$. Las vaquillas con mayores ganancias diarias de peso exhiben incrementos en la frecuencia de descarga de hormona luteinizante (LH) y alcanzaban la pubertad más tempranamente que aquéllas con menores ganancias ${ }^{6}$.

Recibido: 20 mayo 2013 / Aceptado: 26 febrero 2014
Por el contrario, un déficit energético en la dieta de las hembras bovinas retrasa la aparición de la pubertad e incrementa la duración del anestro postparto ${ }^{13}$. El efecto de una deficiencia energética resulta más acentuado en la reproducción, cuando se combina con una deficiencia proteica de la dieta, pues con dietas subproteicas se ve afectado el consumo voluntario, disminuyendo per se el consumo de energía ${ }^{5}$.

Una vaquilla con mayor peso y mayor grado de engrasamiento probablemente tenga un mayor desarrollo genital (escore genital SG) al momento del servicio ${ }^{10}$, el cual puede ser utilizado como predictor de su capacidad de cría ${ }^{11}$. Las vacas de carne con buena condición corporal (CC) en el PP logran tasas de concepción similares a las vaquillas de reposición, una vez que completan la involución uterina y reinician los ciclos ováricos. 
Sin embargo, pueden experimentar infertilidad cuando los requerimientos de nutrientes para mantenimiento y lactancia exceden a los consumidos (vacas de carne PP) o cuando los nutrientes son específicamente particionados y dirigidos hacia la producción láctea (vacas lecheras PP). La subsecuente pérdida de grasa corporal que ocurre en esos casos, afecta negativamente a varios procesos fisiológicos, tornando menos eficiente la reproducción ${ }^{8}$.

Diversos procedimientos han sido propuestos para cuantificar la composición corporal del animal. El método ideal debería ser preciso, repetible y fácil de realizar. Además, debería ser rápido, de bajo costo, aplicable a animales de diferente edad y composición, con alteración mínima (no invasivo) para el animal vivo ${ }^{1}$.

La CC es una herramienta que provee una estimación subjetiva de las reservas corporales del animal (tejido muscular y adiposo) a través de la apreciación visual y táctil ${ }^{7}$. La grasa corporal, a su vez, afecta o al menos está altamente relacionada al fenómeno reproductivo ${ }^{5}$. La ultrasonografía es una técnica reciente, no invasiva de medición de grasa y músculo en animales vivos. Es un método simple y puede ser usado en un gran número de animales a un costo razonable, resultando una alternativa viable para la evaluación de carcasa ${ }^{1}$.

El objetivo del trabajo fue evaluar variables indicativas del desarrollo y composición corporal al inicio del servicio en vaquillas, para ser utilizadas en un modelo matemático que sea predictivo de la tasa de preñez. Además, se analizó si al extender la suplementación en vaquillas retrasadas se logra equiparar su desarrollo y desempeño reproductivo.

\section{MATERIAL Y MÉTODOS}

La experiencia se realizó en el nordeste argentino e incluyó 58 vaquillas Braford criadas y destetadas (otoño del año 2008) en un campo de pastizales de General Obligado (al este de la Provincia de Chaco) y luego trasladadas para su recría a un campo de Colonia Benítez (en la misma provincia). Al inicio de su segundo invierno de recría (año 2009), las vaquillas fueron distribuidas según su peso en 5 lotes $(1: 216 \mathrm{~kg}, 2: 238 \mathrm{~kg}$, 3: $250 \mathrm{~kg}, 4: 270 \mathrm{~kg}$ y $5: 286 \mathrm{~kg}$ ) y el 05/08/09 se inició la suplementación (día 0). Se utilizaron dietas a base de semilla de algodón y sorgo molido con diferente aporte energético y pequeñas variaciones en la proteína bruta durante invierno-primavera, a fin de alcanzar un peso objetivo de entore de alrededor de $320 \mathrm{~kg}$ al inicio de la estación reproductiva. Los lotes fueron mantenidos sobre pasturas tropicales (Grama rhodes y pasto estrella).

Tras 84 días de suplementación, se efectuó una primera selección de las vaquillas para identificar aquéllas que presentaban desarrollo reproductivo adecuado para el servicio. Se evaluó el peso vivo (PV), condición corporal $\left(\mathrm{CC}\right.$; escala de 1 a $9 ; 1=$ emaciada y $9=$ obesa $^{7}$ y por palpación trans-rectal se valoró el tracto reproductivo mediante score genital (SG, escala de Ander- son de 1 a 5) ${ }^{2}$, complementado con ecografía ovárica trans-rectal para determinar tamaño folicular y presencia de cuerpo lúteo (CL).

Asimismo se determinó perímetro torácico (PT, $\mathrm{cm})$ y altura a la grupa (ALT, $\mathrm{cm}$ ) utilizando una cinta métrica inextensible, y 3 medidas corporales ecográficas: grasa subcutánea dorsal (GD, mm), área de ojo de bife $\left(\mathrm{AOB}, \mathrm{cm}^{2}\right)$ y grasa subcutánea en la grupa $(\mathrm{P} 8$, $\mathrm{mm})$.

La medición ecográfica se realizó con un ecógrafo Falco (Pie Medical). Para determinar GD y AOB en el músculo longíssimus dorsi se utilizó un transductor de $17 \mathrm{~cm}$ y $3,5 \mathrm{Mhz}$, mientras que para la determinación de P8 se empleó transductor de 6-8 Mhz. Las mediciones se efectuaron utilizando el software disponible en dicho ecógrafo.

Todas las determinaciones fueron realizadas del lado izquierdo del animal. El AOB y la GD se determinaron entre la $12^{\mathrm{da}}$ y $13^{\text {ra }}$ costilla, transversalmente a la columna vertebral. La GD se midió a $1 / 3$ de distancia del límite medial del músculo longissimus dorsi. El P8 fue evaluado a nivel de la grupa, en el punto de intersección del músculo gluteus medius y el músculo biceps femoris ${ }^{12}$.

Las vaquillas que completados 84 días de suplementación alcanzaron un $\mathrm{SG} \geq 4$ (en adelante llamadas grupo A y consideradas como aptas), se sometieron a un protocolo de sincronización de celo e inseminación artificial a tiempo fijo (IATF; D 84). El protocolo utilizado fue el siguiente: día 84 colocación de dispositivo intravaginal bovino (DIB) conteniendo $0,5 \mathrm{~g}$ progesterona e inyección IM de $2 \mathrm{mg}$ benzoato de estradiol; día 91: retiro e inyección IM de $500 \mu \mathrm{g}$ de un análogo de prostaglandina $\mathrm{F} 2 \alpha ; 0,5 \mathrm{mg}$ cipionato de estradiol y 400 UI de eCG con IATF a las 54 hs post-retiro. Todos los fármacos utilizados fueron del laboratorio Syntex.

En las vaquillas con $\mathrm{SG} \leq 3$ (lote 1 completo $\mathrm{y}$ vaquillas remanentes de otros lotes, en adelante llamadas grupo B), se continuó la suplementación durante 24 días más, completando 108 días a fin de alcanzar un desarrollo reproductivo adecuado para el servicio. En ese momento se registraron idénticas variables pre-servicio y fueron sometidas al mismo protocolo de IATF (D 108).

A los 10 días de la IATF las vaquillas fueron sometidas a servicio natural con dos toros por el lapso aproximado de 90 días. Se realizó el diagnóstico ecográfico de gestación a los 35 días de cada IATF y a los 60 días de finalizado el servicio natural.

Se realizaron análisis estadísticos para verificar si al extender la suplementación en el grupo B se conseguía llevar a las vaquillas a condiciones adecuadas para iniciar una IATF. Para ello se efectuó una comparación de medias de las variables cuantitativas a los 84 y 108 días de suplementación mediante una prueba $t$ para datos pareados. Los porcentajes de preñez fueron analizados bajo distribución de $X^{2}$.

En segunda instancia, se ajustó un modelo de regresión logística donde la variable respuesta Y presentó 
solo valores 1 y 0 (vaquilla preñada / vaquilla no preñada; respectivamente). El modelo para predecir Y, incluyó todas las variables que fueron registradas pre-IATF como variables independientes (PV, CC, SG, ALT, PT, P8, GD, AOB).

Los parámetros del modelo se estimaron utilizando el principio de máxima verosimilitud mediante el uso del módulo "regresión logística" del paquete estadístico Infostat versión 9.0.

\section{RESULTADOS Y DISCUSIÓN}

En la Tabla 1 se muestran los resultados obtenidos para cada variable de los grupos A y B. El grupo B alcanzó los promedios de $320 \mathrm{~kg}$ para $\mathrm{PV}$ y de 3,8 para el SG al extender la suplementación 24 días; siendo estadísticamente diferente de lo observado con 84 días de suplementación $(\mathrm{p}<0,05)$. Todas los valores resultaron diferentes al extender la suplementación en el grupo $\mathrm{B}$, excepto aquéllos correspondientes a las variables de engrasamiento (GD y P8).

Tanto el valor de preñez logrado con la IATF, como el alcanzado con el repaso con toros, resultaron no-diferente entre grupos (Tabla 2). Bajo las condiciones de este ensayo, la estrategia de continuar suplementando las vaquillas resultó válida para alcanzar un peso vivo adecuado, el cual es coincidente con un completo desarrollo reproductivo en la vaquilla (SG 4 y 5).

El SG es utilizado para determinar pubertad en vaquillas y para estimar la posterior capacidad de cría de las mismas ${ }^{11}$. Otros autores obtuvieron 7,6\%; 32,3\% y $32,6 \%$ de preñez a la IATF en vaquillas acebuzadas con $\mathrm{SG}$ de $\leq 2 ; 3 \mathrm{y} \geq 4$ respectivamente ${ }^{9}$. El porcentaje de preñez entre el grupo con $\mathrm{SG}$ de $\leq 2$ fue significativamente diferente al 3 y 4 . Estos porcentajes resultan inferiores a los alcanzados en el presente estudio, ya que en las vaquillas de esta experiencia con un SG prome-

Tabla 1. Resultados obtenidos en los grupos A ( 84 días), B (84 y 108 días de suplementación), $\overline{\mathrm{x}} \pm \mathrm{EE}$ y comparación de medias entre B-84 y B-108.

\begin{tabular}{lccc}
\hline variable & A- 84 & B- 84 & B-108 \\
\hline SG (score) & $4,5 \pm 0,1$ & $2,9 \pm 0,2 \mathrm{a}$ & $3,8 \pm 0,2 \mathrm{~b}$ \\
PV (kg) & $326,5 \pm 4,7$ & $309,1 \pm 8,3 \mathrm{a}$ & $320,1 \pm 7,1 \mathrm{~b}$ \\
CC $(1 \mathrm{a} 9)$ & $4,6 \pm 0,1$ & $4,5 \pm 0,1 \mathrm{a}$ & $4,7 \pm 0,1 \mathrm{~b}$ \\
ALT $(\mathrm{cm})$ & $123,4 \pm 0,6$ & $122,8 \pm 0,8 \mathrm{a}$ & $124,4 \pm 0,8 \mathrm{~b}$ \\
PT $(\mathrm{cm})$ & $159,8 \pm 0,8$ & $155,5 \pm 1,2 \mathrm{a}$ & $157,5 \pm 1,1 \mathrm{~b}$ \\
AOB $\left(\mathrm{cm}^{2}\right)$ & $47,0 \pm 1,1$ & $43,0 \pm 1,4 \mathrm{a}$ & $45,1 \pm 1,0 \mathrm{~b}$ \\
GD $(\mathrm{mm})$ & $3,7 \pm 0,1$ & $3,4 \pm 0,1 \mathrm{a}$ & $3,4 \pm 0,2 \mathrm{a}$ \\
P8 (mm) & $4,9 \pm 0,2$ & $4,0 \pm 0,2 \mathrm{a}$ & $4,4 \pm 0,2 \mathrm{a}$ \\
\hline
\end{tabular}

A-84: grupo A con 84 días de suplementación, B-84: grupo B con 84 días de suplementación, B-108: grupo B con 108 días de suplementación. SG: score genital, PV: peso vivo, CC: condición corporal, ALT: altura a la cadera, PT: perímetro torácico, AOB: área de ojo de bife, GD: grasa subcutánea dorsal, P8: grasa subcutánea de grupa. Letras diferentes entre columnas indican diferencias significativas.
Tabla 2. Porcentajes de preñez a la IATF y al final ( 90 días de servicio) en vaquillas Braford suplementadas durante 84 (grupo A) y 108 días (grupo B).

\begin{tabular}{lccc}
\hline grupo & $\mathrm{n}$ & \% preñez a la IATF & \% preñez final \\
\hline $\mathrm{A}$ & 33 & $45,4(15 / 33)$ & $81,8(27 / 33)$ \\
$\mathrm{B}$ & 25 & $56,0(14 / 25)$ & $80,0(20 / 25)$ \\
\hline
\end{tabular}

IATF: inseminación artificial a tiempo fijo.

Tabla 3. Resultados del análisis de regresión logística utilizando como variables de ajuste el score genital y la altura a la cadera para predecir preñez a la IATF.

\begin{tabular}{lcc}
\hline vaquillas & vacías predichas & preñadas predichas \\
\hline vacías observadas & 20 & 8 \\
preñadas observadas & 10 & 19 \\
\hline
\end{tabular}

IATF: inseminación artificial a tiempo fijo.

dio de 4,2 se obtuvo alrededor de un $50,7 \%$ de preñez. Sin embargo, es coincidente con lo reportado en otra experiencia en la que informan un $54,0 \%$ de preñez y valores de SG y preñez de $3=40 \%$; $4=54 \%$ y $5=62 \%{ }^{11}$.

Otros investigadores analizaron las variables ultrasonográficas de composición corporal (grasa intramuscular, AOB, GD y P8) en vaquillas Angus pre-servicio e informan valores de P8 y AOB coincidentes con ciclicidad de 6,6 a $7,4 \mathrm{~mm}$ y de $64,3 \mathrm{~cm}^{2}$; respectivamente ${ }^{10}$. Estos valores son sustancialmente superiores a los hallados en las vaquillas acebuzadas del presente trabajo (4,5 $\mathrm{mm}$ y $46 \mathrm{~cm}^{2}$; respectivamente). Sin embargo, no encontraron una relación significativa entre GD o grasa intramuscular con ciclicidad o con porcentaje de preñez. Es interesante señalar que estos autores comprobaron una relación entre SG y P8, pero no con GD. Esto es atribuido a que el P8 es una medida con mayor heredabilidad y repetibilidad que la GD. Además existe mayor variación en animales jóvenes y en animales flacos en la GD que en el P8.

De todas las variables registradas en las vaquillas del presente ensayo (PV, CC, SG, ALT, PT, AOB, GD y P8), solamente SG y ALT lograron un ajuste del modelo de regresión logística, que fue realizada utilizando el SG pre-servicio y la altura a la grupa como variables regresoras, el grupo como variable clasificadora y la respuesta dicotómica preñada / no preñada como variable dependiente. Dicha regresión se efectuó sobre un total de 57 observaciones, ya que por dato faltante una de ellas fue eliminada del análisis.

En la Tabla 3 se informan las asignaciones observadas y predichas por el modelo. La regresión logística permitió predecir con exactitud el resultado del servicio en el 68\% de los casos (39/57), asignando el evento preñez (éxito) a aquellas vaquillas que presentaron un valor de altura a la grupa que no superó los $122 \mathrm{~cm}$ en combinación con un SG de 5.

Otros investigadores demostraron la existencia de una correlación desfavorable entre altura a la grupa (variable de crecimiento) y edad a la pubertad (variable 
de reproducción) en vaquillas Brahman ${ }^{14}$. En dichas vaquillas, la selección por aumento en la altura a los 18 meses de edad resulta en vaquillas más altas y de mayor edad a la pubertad. La selección por crecimiento dado por aumento de masa muscular no tendría un efecto perjudicial sobre la edad a la pubertad en vaquillas ${ }^{15}$.

En otro trabajo, al analizar la relación de la pubertad y el crecimiento en el ganado, se concluyó que las diferencias en el potencial genético de crecimiento por aumento de peso no afectaban la edad de la pubertad de manera significativa ${ }^{3}$. Sin embargo, después de ajustar por raza-tipo y manejo, las vaquillas más altas a los 315 días de edad alcanzaron la pubertad a mayor edad con un coeficiente de regresión de $2,04 \mathrm{~d} / \mathrm{cm}$. Tales resultados indican que la mayor variación entre razas-tipos genéticos fue debida a la altura y conducen a concluir acerca de la necesidad de considerar todos los caracteres de la pubertad en forma simultánea con el fin de tener una comprensión completa de sus relaciones.

La información presentada, en coincidencia con los resultados de nuestro análisis, muestra que la altura y el score genital son las variables más adecuadas para ser utilizadas como predictoras de preñez en vaquillas. El peso vivo está asociado positivamente con el score genital. El modelo desarrollado permite predecir con una exactitud del $68 \%$ el resultado del servicio en las vaquillas (preñada o vacía). En las condiciones en que se desarrolló esta experiencia, las vaquillas no deben superar los $122 \mathrm{~cm}$ de altura a la grupa y presentar un score genital de 5 al inicio de la estación reproductiva para lograr la mayor probabilidad de preñez.

\section{REFERENCIAS}

1. Aass L, Fristedt CG, Gresham JD. 2009. Ultrasound prediction of intramuscular fat content in lean cattle. Livestock Sci 125: 177-186.

2. Anderson KJ, LeFever DG, Brinks SJ, Odde KG. 1991. The use of reproductive tract scoring in beef heifers. AgriPractice 12: 19-26.

3. Baker JF, Stewart TS, Long CR, Cartwright TC. 1988. Multiple regression and principal components analysis of puberty and growth in cattle. J Anim Sci 66: 2147-2158.

4. Day ML, Imakawa K, Zalesky DD, Kittok RJ, Kinder JE. 1986. Effects of dietary energy intake during the prepuberal period on secretion of luteinizing hormone and responsiveness of the pituitary to luteinizing-hormone releasing hormone in heifers. J Anim Sci 62: 1641-1648.
5. Dunn TG, Moss GE. 1992. Effects of nutrient deficiencies and excesses on reproductive efficiency of livestock. $J$ Anim Sci 70: 1580-1593.

6. Hall JB, Schillo KK, Fitzgerald BP, Bradley NW. 1994. Effects of recombinant bovine somatotropin and dietary energy intake on growth, secretion of luteinizing hormone, follicular development, and onset of puberty in beef heifers. J Anim Sci 72: 709-718.

7. Herd DB, Sprott LR. 1986. Body condition, nutrition and reproduction in beef cows. Bulletin Agricultural Extension Service, Texas A\&M University, $\mathrm{N}^{\mathrm{o}} 1526,11 \mathrm{p}$.

8. Lucy MC. 2003. Mechanisms linking nutrition and reproduction in postpartum cows. Reproduction 61: 415-427.

9. McKinniss EN, Esterman RD, Woodall SA, Austin BR, Hersom MJ, Thatcher WW, Yelich JV. 2011. Evaluation of two progestogen-based estrous synchronization protocols in yearling heifers of Bos indicus x Bos taurus breeding. Theriogenology 75: 1699-1707.

10. Minick JA, Wilson DE, Rouse GH, Hassen A, Sealock MP, Hopkins S. 2001. Relationship between body composition and reproduction in heifers. Iowa State University (Ames), Beef Research Report R. 1769.

11. Pence M, Bredahl R, Thomson JU. 1999. Clinical use of reproductive tract scoring to predict pregnancy outcome. Iowa State University (Ames), Beef Research Report R. 1656.

12. Realini CE, Williams RE, Pringle TD, Bertrand JK. 2001. Gluteus medious rump fat depths as additional live animal ultrasound measurements for predicting retail product and trimmable fat in beef carcasses. J Anim Sci 79: 1378-1385.

13. Short RE, Bellows RA, Staigmiller RB, Berardinelli JG, Custer EE. 1990. Physiological mechanisms controlling anestrus and infertility in postpartum beef cattle. $J$ Anim Sci 68: 799-816.

14. Vargas CA, Elzo MA, Chase CC, Chenoweth PJ, Olson TA. 1998. Estimation of genetic parameters for scrotal circumference, age at puberty in heifers, and hip height in Brahman cattle. J Anim Sci 76: 2536-2541.

15. Wolfe MW, Stumpf TT, Wolfe PL, Day ML, Koch RM, Kinder JE. 1990. Effect of selection for growth traits on age and weight at puberty in bovine females. J Anim Sci 68: $1595-1602$. 